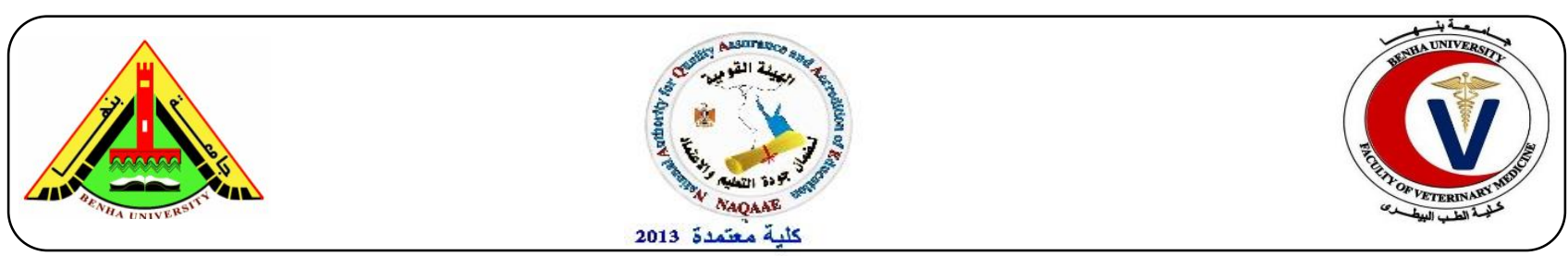

\title{
Biochemical role of zinc oxide and propolis nanoparticles in protection rabbits against coccidiosis
}

Afaf D. Abd El Megid ${ }^{1}$, Mohamed Khaled ${ }^{1}$, Mahmoud Abdeleghaffar Emam², Amira Adel ${ }^{\mathbf{1}}$

${ }^{I}$ Department of Biochemistry. Faculty of Veterinary Medicine, Benha University.

${ }^{2}$ Department of of Histology. Faculty of Veterinary Medicine, Benha University.

\section{A B S T R A C T}

Coccidiosis is a protozoan infection of animals which causes growth retardation and high mortality in rabbits. There are two forms of coccidiosis were recognized in rabbits; hepatic and intestinal coccidiosis. The hepatic coccidiosis is due to Eimeria stiedae cause severe damage for liver which leads to death. Nanotechnology is the most important technology in our life where the use of nanotechnology in medicine offers some exciting possibilities. So, this study uses zinc oxide nanoparticles as chemical therapy and propolis nanoparticles as natural therapy. Twenty male rabbits aged 1 - 2 months and weighting 1250 - 1400 gm was used. Animals were divided into 4 groups (5 animals per each): control negative group; uninfected male rabbits and saved healthy, control positive group; infected male rabbits by 40.000 sporulated oocyst of Eimeria spp. and not treated. Zinc oxide nanoparticles treated group; infected male rabbits by 40.000 sporulated oocyst of Eimeria spp. and treated orally with $10 \mathrm{mg} \mathrm{ZnO-NPs/Kg}$ body weight daily for 5 consecutive days. Propolis nanoparticles treated group; infected male rabbits by 40.000 sporulated oocyst of Eimeria spp. and treated orally with $100 \mathrm{mg}$ propolis-NPs/Kg body weight daily for 7 consecutive days. Serum ALT, CRP, zinc, cholinesterase, plasma GR, SOD and CAT were measured in addition to; histopathological examination of liver was done. The results indicated that the serum ALT and CRP were increased but serum cholinesterase, zinc, plasma GR, SOD and CAT were decreased in infected group, with treatment all parameters were improved, also examination of histological sections of livers from all treated groups did not detect any protozoal stage in their parenchyma nor bile ducts. Also, no or minimal papillary projection of epithelium of bile duct with decrease of peribiliary fibrosis could be seen in comparison control positive group. In conclusion, zinc oxide nanoparticles and propolis nanoparticles play a protective role against Eimeria infestation in rabbits.

Keywords: Coccidiosis, Nanotechnology, Propolis-NPs, ZnO-NPs.

(http://www.bvmj.bu.edu.eg)

(BVMJ-34(1): 314-328, 2018)

\section{INTRODUCTION}

Coccidiosis is a protozoan infection of animals which causes growth retardation and high mortality in rabbits (El-Akabawy et al., 2004). There are two forms of coccidiosis were recognized in rabbits; hepatic and intestinal coccidiosis. The hepatic coccidiosis is due to Eimeria stiedae cause severe damage for liver which led to death (Oncel et al., 2011). The characteristic symptoms associated with hepatic coccidiosis in rabbits include loss of appetite, anorexia, hair loss, diarrhea, yellowish mucous 
membranes, fatigue, abdomen swelling and significant loss in body weight (AlMathal ,2010), as well as increase in serum AST, ALT , GGT and L-MDA but decrease erythrocyte CAT and SOD activities which have been reported in the rabbits with hepatic coccidiosis (Cam et al. , 2008).

Nanotechnology is the most important technology in our life where the use of nanotechnology in medicine offers some exciting possibilities. Now, Researchers try to use more effective drugs against coccidia as nanoparticles because nano medicine has recently emerged as a better option for the treatment of various diseases and researches into nano technological approache to infectious diseases increases quickly (Allahverdiyev et al ., 2011). There is more expectation that nano particles will be able to be used in the treatment of various diseases in the future (Angeli et al., 2008). In this study, we used zinc oxide nano particles due to zinc supplementation have been successfully used as a therapeutic and preventive agent for many conditions (Prasad, 2004). ZnO-NPs have effective antibacterial activity and antimicrobial efficacy against various bacterial and fungal pathogens (Gunalan et al., 2012). Zinc oxide nanoparticles have anticoccidial properties (Dkhil et al., 2015). Dietary supplementation with low dose of zinc oxide nanoparticles improved growth performance and intestinal morphology, reducing diarrhea and intestinal inflammatory in weaning piglets (Long et al., 2017). And we used propolis nanoparticles because propolis has many biological and pharmacological properties (Bufalo et al., 2009). Propolis (blue glue) is a natural nontoxic resinous sticky substance with very complex chemical composition produced by mixing secretions of honey bees secretions hypopharyngeal glands with the digested product of resins collected from plant particularly from flowers of plants, leaf buds and the bark of trees (Coutinho, 2012). Propolis extracts from Serbis has antioxidant activity (Potkonjak et al., 2012). Propolis-NPs have antimicrobial activity against Escherichia coli and Staphylococcus aureus (Rangasamy et al., 2012). Propolis-NPs have antibacterial and antifungal activity against Staphilocuccus aureus and Candida albicans where it is used efficiently in controlling of bacterial and fungal diseases (Afrouzan et al., 2012). Propolis-NPs was effective in the treatment of diabetes due to the reduction of blood sugar level and the regeneration of damaged $\beta$-cells observed in streptozotocininduced diabetic rats (Nyun-Ki et al., 2010). So, this study investigated the potential protective role of zinc oxide nanoparticles and propolis nanoparticles against Eimeria infestation in rabbits.

\section{Materials and methods}

\subsection{Experimental animals:}

A total number of 20 animals male rabbits divided into 4 groups each group contain 5 rabbits weighting $1250-1400 \mathrm{gm}$ and aged 1 - 2 months old were used for the experimental investigation of this study. Rabbits were housed separately in clean wire floored cages in the lab. They were kept 12 days after setting up the groups before beginning the experiment for adaptation with the environment. The rabbits were fed on diet in the form of pillets obtained from $\mathrm{El}$ - Baraka Company in Cairo, Egypt. This diet composed of $58 \%$ Carbohydrate, $20 \%$ Protein, $4.0 \%$ Lipid, $3.0 \%$ Cellulose, $3.0 \%$ (Minerals, Calcium, Phosphorous) $12 \%$ Moisture.

\subsection{Drugs:-}

Propolis-NPs: Natural propolis was obtained from Imtenan Pharma, Cairo, Egypt. Then, it was converted into propolis-NPs by grinding in RTZ-400 for 3 hours by dry pall milling mechanism till reach to size $100 \mathrm{~nm}$. In 
Egyptian Petroleum Research Institute in Naser city, Cairo, Egypt.

ZnO-NPs: Zinc oxide was obtained from Biostc Company, Cairo, Egypt. Then, it was converted into ZnO-NPs by grinding in RTZ400 for 3 hours by dry pall milling mechanism till reach to size $100 \mathrm{~nm}$. In Egyptian Petroleum Research Institute in Naser city, Cairo, Egypt.

\subsection{Dose of sporulated oocysts:}

The dose of sporulated oocyst was calculated according to (Long et al., 1976). Where each rabbit was given $1 \mathrm{ml}$ under the tongue (Coudert et al., 2000) containing $4 \times 10 * 4$ sporulated oocyst of Eimeria spp. using Mc Master Technique (Fisher and Kelley, 1977).

\subsection{Animal grouping:}

Rabbits were divided into 4 groups:-

Group 1: (Control negative group) contained 5 uninfected male rabbits and saved healthy.

Group 2: (Control positive group) contained 5 infected male rabbits by 40.000 sporulated oocyst of Eimeria spp. and not treated.

Group 3: (ZnO-NPs treated group) contained 5 infected male rabbits by 40.000 sporulated oocyst of Eimeria spp. and treated orally with $10 \mathrm{mg}$ nano $\mathrm{ZnO}-\mathrm{NPs} / \mathrm{Kg}$ body weight body weight daily for 5 consecutive days (Dkhil et al., 2015).

Group 4: (Propolis-NPs treated group) contained 5 infected male rabbits by 40.000 sporulated oocyst of Eimeria spp. and treated orally with $100 \mathrm{mg}$ propolis nanoparticles / $\mathrm{Kg}$ body weight body weight daily for 7 consecutive days (Rangasamy et al., 2012).

\subsection{Biochemical investigations:}

Blood samples were collected after overnight fasting from all animals from the marginal ear vein of all rabbits for making serum alanine aminotransferase (ALT) determination according to the method described by (Henry, 1974) using the kit supplied by BIOSTC company , Egypt . Serum CRP was determined according to the method described by (Macleod and Avery, 1941) using the kit supplied by BIOSTC company, Egypt. Serum zinc was determined according to the method described by (Johnsen and Eliasson., 1987) using the kit supplied by Salucea, BioSTC company, Egypt. Serum cholinesterase (CHE) was determined according to the method described by (Szasz, 1968) using the kit supplied by BEN, BIOSTC company, Egypt. Plasma glutathione reductase (GR) was determined according to the method described by (Goldberg and Spooner., 1983) using the kit supplied by Biodiagnostic, company, Egypt. Plasma SOD activity was determined according to the method described by (Nishikimi et al., 1972).Plasma catalase (CAT) was determined according to the method described by (Aebi, 1984) using the kit supplied by Biodiagnostic, company, Egypt. We measured all parameters before treatment and one week, two weeks, three weeks post treatment.

\subsection{Histopathological specimens:}

At the end of experiment, all rabbits were decapitated. Livers of all rabbits were thoroughly examined for the gross lesions and liver tissues specimens were collected in $10 \%$ neutral buffer formalin. After fixation of liver specimens in $10 \%$ neutral buffer formalin, dehydration in ascending grades of alcohol, clearance in xylene were done. Finally, liver specimens were embedded in paraffin. Paraffin sections of $5 \mu \mathrm{m}$ were cut and stained for $\mathrm{H} \& \mathrm{E}$ as outlined by (Bancroft and Marilyne, 2008).

\section{RESULTS}

Infection with Eimeria spp. to normal rabbits exhibited a significant increase in serum ALT activity and serum CRP concentration but exhibited a significant decrease in serum zinc 
concentration, serum cholinesterase activity, plasma GR, SOD and CAT activities before treatment, one week, two weeks and three weeks post treatment compared to control negative group but with administration of ZnO-NPs and propolis-NPs to infected rabbits after 2 weeks of infection exhibited a significant decrease in serum ALT activity and serum CRP concentration but exhibited a significant increase in serum zinc concentration, serum cholinesterase activity, plasma GR, SOD and CAT activities.

\section{Histopathological results:}

\section{Liver of control negative group:}

Histological sections showed normal feature of liver structure; normal central vein, and hepatocytes were arranged in cords and separated by blood sinusoid (Fig.1).

\section{Liver of control positive group:}

Sections through the lesions of liver from this group showed the different protozoal stages including microgametocytes, macrogametocyts and oocyts that were separated from hepatic parenchyma by thick connective tissue capsule (Figs.2). Hyperplasia of biliary columnar epithelium with papillary projections in addition to, peribiliary fibrosis with infiltration of mononuclear cells was also common features in the bile ducts (Fig.3). Within the hepatic parenchyma, congestion of central vein and sinusoids (Fig.4) and focal mononuclear cells infiltration (Fig.5) could be observed.

\section{Liver of treated groups:}

Examination of histological sections of livers from treated groups did not detect any protozoal stage in their parenchyma or bile ducts. Congestion of the central vein, blood sinusoid and hepatic vein was the common feature for livers from $\mathrm{ZnO}-\mathrm{NPs}$ (6) and propolis-NPs (8) treated groups. In $\mathrm{ZnO}-\mathrm{NPs}$ treated group, no papillary projection of biliary duct epithelium with obvious decrease of peribiliary fibrosis were identified (Fig.7) in comparison to control positive group. In propolis-NPs treated group, liver showed hyperplasia of epithelium of bile duct with fibrous coat (Fig.9).

Table 1: Serum ALT activity (U/L) of experimentally infected male rabbits with Eimeria spp. before and after treatment with $\mathrm{ZnO}-\mathrm{NPs}$, propolis-NPs (mean $\pm \mathrm{SE}$ ).

\begin{tabular}{|c|c|c|c|c|c|}
\hline \multirow[b]{2}{*}{ Groups } & \multicolumn{4}{|l|}{ Period } & \multirow[b]{2}{*}{ Mean } \\
\hline & $\begin{array}{l}\text { Before } \\
\text { treatment }\end{array}$ & $\begin{array}{l}\text { One week post } \\
\text { treatment }\end{array}$ & $\begin{array}{l}\text { Two weeks } \\
\text { post treatment }\end{array}$ & $\begin{array}{l}\text { Three weeks } \\
\text { post treatment }\end{array}$ & \\
\hline $\begin{array}{l}\text { Control } \\
\text { (Healthy) }\end{array}$ & $34.40 \pm 1.36^{\mathrm{dA}}$ & $34.57 \pm 0.45^{\mathrm{cA}}$ & $33.77 \pm 0.61^{\mathrm{dA}}$ & $32.93 \pm 1.34^{\mathrm{dA}}$ & $33.92 \pm 0.48^{\mathrm{e}}$ \\
\hline $\begin{array}{l}\text { Control } \\
\text { (Infected, } \\
\text { treated) }\end{array}$ & $93.40 \pm 4.83^{\mathrm{aC}}$ & $106.97 \pm 4.24^{\mathrm{aA}}$ & $103.70 \pm 2.70^{\mathrm{aB}}$ & $92.63 \pm 1.42^{\mathrm{aC}}$ & $99.18 \pm 2.42^{\mathrm{a}}$ \\
\hline $\begin{array}{l}\text { Exp. infected and } \\
\text { ZnO-NPs treated }\end{array}$ & $78.07 \pm 4.88^{\mathrm{bA}}$ & $30.57 \pm 1.87^{\mathrm{dB}}$ & $30.20 \pm 0.64^{\mathrm{eB}}$ & $31.17 \pm 1.32^{\mathrm{deB}}$ & $42.50 \pm 6.30^{\mathrm{c}}$ \\
\hline $\begin{array}{l}\text { Exp. infected and } \\
\text { propolis-NPs treated }\end{array}$ & $74.23 \pm 0.81^{\mathrm{cA}}$ & $40.77 \pm 0.73^{\mathrm{bC}}$ & $41.77 \pm 0.66^{\mathrm{bC}}$ & $44.37 \pm 0.47^{\mathrm{bB}}$ & $50.28 \pm 4.20^{\mathrm{b}}$ \\
\hline
\end{tabular}

a, b \& c: There is a significant difference $(\mathrm{P}<0.05)$ between any two means, within the same column have the different superscript letter 
.A, B \& C: There is a significant difference $(\mathrm{P}<0.05)$ between any two means, within the same row have the different superscript letter.

Table 2: Serum CRP concentration $(\mathrm{mg} / \mathrm{L})$ of experimentally infected male rabbits with Eimeria spp. before and after treatment with with $\mathrm{ZnO}-\mathrm{NPs}$, propolis-NPs (mean $\pm \mathrm{SE}$ ).

\begin{tabular}{|c|c|c|c|c|c|}
\hline \multirow[b]{2}{*}{ Groups } & \multicolumn{4}{|l|}{ Period } & \multirow[b]{2}{*}{ Mean } \\
\hline & $\begin{array}{l}\text { Before } \\
\text { treatment }\end{array}$ & $\begin{array}{l}\text { One week } \\
\text { post } \\
\text { treatment }\end{array}$ & $\begin{array}{l}\text { Two weeks } \\
\text { post } \\
\text { treatment }\end{array}$ & $\begin{array}{l}\text { Three weeks } \\
\text { post } \\
\text { treatment }\end{array}$ & \\
\hline $\begin{array}{l}\text { Control (-ve) } \\
\text { (Healthy) }\end{array}$ & $\begin{array}{l}23.00 \pm 0.58^{e} \\
\text { B }\end{array}$ & $\begin{array}{l}22.67 \pm 0.88^{d} \\
B\end{array}$ & $\begin{array}{l}23.33 \pm 0.88^{\mathrm{e}} \\
\text { B }\end{array}$ & $\begin{array}{l}28.00 \pm 0.58^{d} \\
A\end{array}$ & $\begin{array}{l}24.25 \pm 0.7 \\
3^{\mathrm{e}}\end{array}$ \\
\hline $\begin{array}{l}\text { Control (+ve) } \\
\text { (Infected, non } \\
\text { treated) }\end{array}$ & $\begin{array}{l}192.33 \pm 1.45 \\
b D\end{array}$ & $\begin{array}{l}200.67 \pm 1.45 \\
\mathrm{aC}\end{array}$ & $\begin{array}{l}204.33 \pm 2.73 \\
\mathrm{aB}\end{array}$ & $\begin{array}{l}217.67 \pm 4.33 \\
\text { aA }\end{array}$ & $\begin{array}{l}203.75 \pm 3 . \\
00^{\mathrm{a}}\end{array}$ \\
\hline $\begin{array}{l}\text { Exp. infected } \\
\text { and ZnO-NPs } \\
\text { treated }\end{array}$ & $\begin{array}{l}195.00 \pm 5.03 \\
\text { bA }\end{array}$ & $\begin{array}{l}22.67 \pm 0.88^{d} \\
B\end{array}$ & $\begin{array}{l}23.67 \pm 0.88^{e} \\
\text { B }\end{array}$ & $\begin{array}{l}25.00 \pm 1.00^{\mathrm{e}} \\
\mathrm{B}\end{array}$ & $\begin{array}{l}66.58 \pm 22 . \\
38^{\mathrm{d}}\end{array}$ \\
\hline $\begin{array}{l}\text { Exp. infected } \\
\text { and propolis- } \\
\text { NPs treated }\end{array}$ & $\begin{array}{l}200.00 \pm 3.46 \\
\text { aA }\end{array}$ & $\begin{array}{l}51.33 \pm 1.76^{b} \\
C\end{array}$ & $\begin{array}{l}56.67 \pm 1.86^{b} \\
\text { B }\end{array}$ & $\begin{array}{l}58.67 \pm 1.76^{b} \\
\text { B }\end{array}$ & $\begin{array}{l}91.67 \pm 18 . \\
90^{\mathrm{b}}\end{array}$ \\
\hline
\end{tabular}

a, b \& c: There is a significant difference $(\mathrm{P}<0.05)$ between any two means, within the same column have the different superscript letter.

A, B \& C: There is a significant difference $(\mathrm{P}<0.05)$ between any two means, within the same row have the different superscript letter.

Table 3: Serum zinc concentration $(\mu \mathrm{g} / \mathrm{dl})$ of experimentally infected male rabbits with Eimeria spp. before and after treatment with with $\mathrm{ZnO}-\mathrm{NPs}$, propolis-NPs (mean $\pm \mathrm{SE}$ ).

\begin{tabular}{|c|c|c|c|c|c|}
\hline \multirow[b]{2}{*}{ Groups } & \multicolumn{4}{|l|}{ Period } & \multirow[b]{2}{*}{ Mean } \\
\hline & $\begin{array}{l}\text { Before } \\
\text { treatment }\end{array}$ & $\begin{array}{l}\text { One week } \\
\text { post treatment }\end{array}$ & $\begin{array}{l}\text { Two weeks } \\
\text { post treatment }\end{array}$ & $\begin{array}{l}\text { Three weeks } \\
\text { post } \\
\text { treatment }\end{array}$ & \\
\hline $\begin{array}{l}\text { Control } \\
\text { (Healthy) }\end{array}$ & $84.73 \pm 2.22^{\mathrm{aB}}$ & $86.63 \pm 1.72^{\mathrm{dA}}$ & $86.60 \pm 2.25^{\mathrm{cA}}$ & $88.17 \pm 2.31^{\mathrm{cA}}$ & $86.53 \pm 0.98^{b c}$ \\
\hline $\begin{array}{l}\text { Control (+ve) } \\
\text { (Infected, non treated) }\end{array}$ & $76.93 \pm 1.00^{\mathrm{bA}}$ & $74.67 \pm 0.64^{\mathrm{eBC}}$ & $75.60 \pm 0.35^{\mathrm{eAB}}$ & $73.37 \pm 0.43^{\mathrm{eC}}$ & $75.14 \pm 0.48^{\mathrm{e}}$ \\
\hline $\begin{array}{l}\text { Exp. infected and } \\
\text { ZnO-NPs treated }\end{array}$ & $76.00 \pm 1.45^{\mathrm{bC}}$ & $100.27 \pm 0.20^{\mathrm{aA}}$ & $99.33 \pm 0.54^{\mathrm{aAB}}$ & $98.43 \pm 0.83^{\mathrm{aB}}$ & $93.51 \pm 3.08^{\mathrm{a}}$ \\
\hline $\begin{array}{l}\text { Exp. infected and } \\
\text { propolis-NPs treated }\end{array}$ & $76.93 \pm 1.17^{\mathrm{bD}}$ & $86.70 \pm 1.97^{\mathrm{dA}}$ & $82.90 \pm 0.98^{\mathrm{dB}}$ & $80.40 \pm 0.53^{\mathrm{dC}}$ & $81.73 \pm 1.20^{\mathrm{d}}$ \\
\hline
\end{tabular}

$\mathrm{a}, \mathrm{b} \& \mathrm{c}$ : There is a significant difference $(\mathrm{P}<0.05)$ between any two means, within the same column have the different superscript letter. A, B \& $\mathrm{C}$ : There is a significant difference $(\mathrm{P}<0.05)$ between any two means, within the same row have the different superscript letter. 
Table 4: Serum cholinesterase activity (U/L) of experimentally infected male rabbits with Eimeria spp. before and after treatment with with $\mathrm{ZnO}-\mathrm{NPs}$, propolis-NPs (mean $\pm \mathrm{SE}$ ).

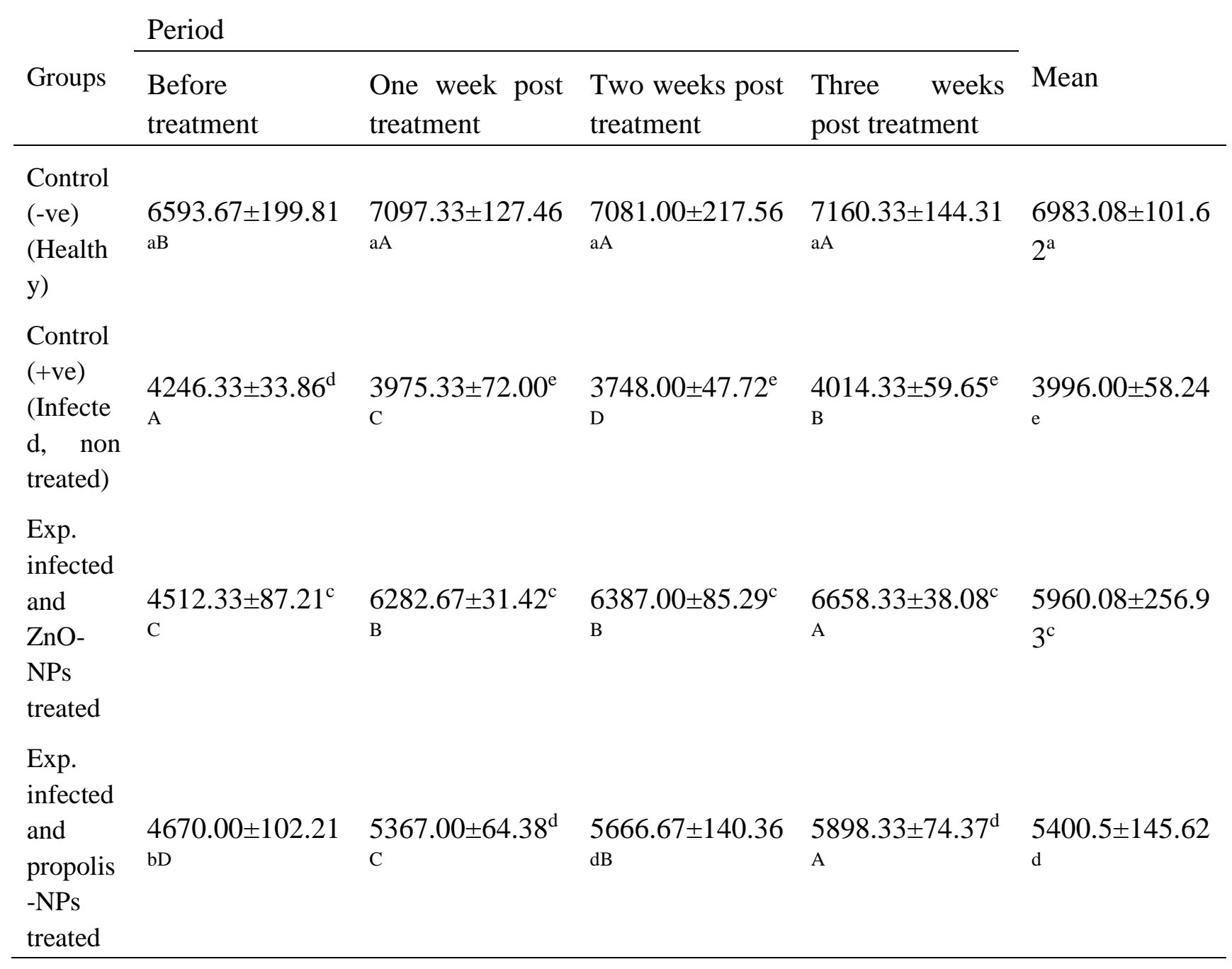

$\mathrm{a}, \mathrm{b} \& \mathrm{c}$ : There is a significant difference $(\mathrm{P}<0.05)$ between any two means, within the same column have the different superscript letter. A, B \& $\mathrm{C}$ : There is a significant difference $(\mathrm{P}<0.05)$ between any two means, within the same row have the different superscript letter.

Table 5: Plasma glutathione reductase activity (U/L) of experimentally infected male rabbits with Eimeria spp. before and after treatment with with ZnO-NPs, propolis-NPs (mean \pm SE).

\begin{tabular}{|c|c|c|c|c|c|c|}
\hline \multirow[b]{2}{*}{ Groups } & & \multicolumn{4}{|l|}{ Period } & \multirow[b]{2}{*}{ Mean } \\
\hline & & $\begin{array}{l}\text { Before } \\
\text { treatment }\end{array}$ & $\begin{array}{l}\text { One week } \\
\text { post } \\
\text { treatment }\end{array}$ & $\begin{array}{l}\text { Two weeks } \\
\text { post } \\
\text { treatment }\end{array}$ & $\begin{array}{l}\text { Three weeks } \\
\text { post treatment }\end{array}$ & \\
\hline $\begin{array}{l}\text { Control } \\
\text { (Healthy) }\end{array}$ & $(-v e)$ & $48.65 \pm 0.23^{\mathrm{aA}}$ & $46.82 \pm 2.46^{\mathrm{aB}}$ & $46.37 \pm 3.05^{\mathrm{aB}}$ & $49.48 \pm 0.32^{\mathrm{aA}}$ & $47.83 \pm 0.92^{\mathrm{a}}$ \\
\hline $\begin{array}{l}\text { Control } \\
\text { (Infected, } \\
\text { treated) }\end{array}$ & $\begin{array}{r}(+\mathrm{ve}) \\
\text { non }\end{array}$ & $29.03 \pm 0.16^{\mathrm{bA}}$ & $28.77 \pm 0.20^{\mathrm{eA}}$ & $28.66 \pm 0.23^{\mathrm{dA}}$ & $28.55 \pm 0.23^{\mathrm{eA}}$ & $28.75 \pm 0.10^{\mathrm{e}}$ \\
\hline \multicolumn{2}{|c|}{$\begin{array}{l}\text { Exp. infected and } \\
\text { ZnO-NPs treated }\end{array}$} & $28.75 \pm 0.17^{\mathrm{bB}}$ & $\begin{array}{r}45.31 \pm 0.27^{\mathrm{bA}} \\
\mu_{19}\end{array}$ & $45.40 \pm 0.10^{\mathrm{aA}}$ & $45.41 \pm 0.23^{\mathrm{bA}}$ & $41.22 \pm 2.17^{\mathrm{b}}$ \\
\hline
\end{tabular}


Exp. infected and

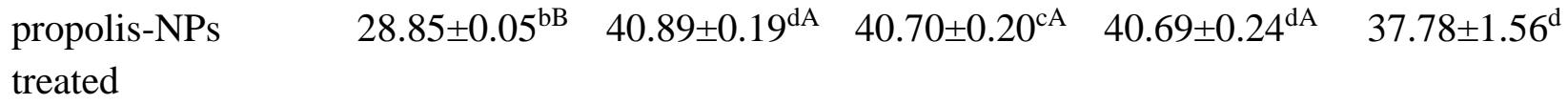

Table 6: Plasma SOD activity (U/ml) of experimentally infected male rabbits with Eimeria spp. before and after treatment with with $\mathrm{ZnO}-\mathrm{NPs}$, propolis-NPs (mean $\pm \mathrm{SE}$ ).

\begin{tabular}{|c|c|c|c|c|c|}
\hline \multirow[b]{2}{*}{ Groups } & \multicolumn{4}{|l|}{ Period } & \multirow[b]{2}{*}{ Mean } \\
\hline & $\begin{array}{l}\text { Before } \\
\text { treatment }\end{array}$ & $\begin{array}{l}\text { One week } \\
\text { post } \\
\text { treatment }\end{array}$ & $\begin{array}{l}\text { Two weeks } \\
\text { post treatment }\end{array}$ & $\begin{array}{l}\text { Three weeks } \\
\text { post } \\
\text { treatment }\end{array}$ & \\
\hline $\begin{array}{l}\text { Control } \\
\text { (Healthy) }\end{array}$ & $44.03 \pm 0.94^{\mathrm{aA}}$ & $44.77 \pm 2.09^{\mathrm{aA}}$ & $44.13 \pm 0.83^{\mathrm{aA}}$ & $42.60 \pm 1.22^{\mathrm{aB}}$ & $43.88 \pm 0.63^{\mathrm{a}}$ \\
\hline $\begin{array}{l}\text { Control } \\
\text { (Infected, } \\
\text { treated) }\end{array}$ & $28.33 \pm 0.98^{\mathrm{bA}}$ & $26.93 \pm 0.80^{\mathrm{fB}}$ & $27.67 \pm 0.84^{\mathrm{eAB}}$ & $22.47 \pm 1.10^{\mathrm{eC}}$ & $26.35 \pm 0.80^{\mathrm{e}}$ \\
\hline $\begin{array}{l}\text { Exp. infected and } \\
\text { ZnO-NPs treated }\end{array}$ & $29.37 \pm 0.74^{\mathrm{bD}}$ & $39.60 \pm 0.36^{\mathrm{cC}}$ & $42.27 \pm 0.55^{\mathrm{bA}}$ & $41.13 \pm 0.83^{\mathrm{bB}}$ & $38.09 \pm 1.57^{\mathrm{b}}$ \\
\hline $\begin{array}{l}\text { Exp. infected and } \\
\text { propolis-NPs } \\
\text { treated }\end{array}$ & $29.23 \pm 1.29^{\mathrm{bD}}$ & $30.53 \pm 0.61^{\mathrm{eC}}$ & $33.70 \pm 0.55^{\mathrm{dA}}$ & $31.80 \pm 1.71^{\mathrm{dB}}$ & $31.32 \pm 0.70^{\mathrm{d}}$ \\
\hline
\end{tabular}

a, b \& c: There is a significant difference $(\mathrm{P}<0.05)$ between any two means, within the same column have the different superscript letter.

A, B \& C: There is a significant difference $(\mathrm{P}<0.05)$ between any two means, within the same row have the different superscript letter.

Table 7: Plasma catalase activity (U/L) of experimentally infected male rabbits with Eimeria spp. before and after treatment with with $\mathrm{ZnO}-\mathrm{NPs}$, propolis-NPs (mean $\pm \mathrm{SE}$ ).

\begin{tabular}{|c|c|c|c|c|c|}
\hline \multirow[b]{2}{*}{ Groups } & \multicolumn{4}{|l|}{ Period } & \multirow[b]{2}{*}{ Mean } \\
\hline & $\begin{array}{l}\text { Before } \\
\text { treatment }\end{array}$ & $\begin{array}{l}\text { One week post } \\
\text { treatment }\end{array}$ & $\begin{array}{l}\text { Two weeks post } \\
\text { treatment }\end{array}$ & $\begin{array}{l}\text { Three weeks } \\
\text { post treatment }\end{array}$ & \\
\hline Control & & & & & \\
\hline $\begin{array}{l}\text { (-ve) } \\
\text { (Healthy } \\
\text { ) }\end{array}$ & $\begin{array}{l}1229.67 \pm 41.80^{\mathrm{a}} \\
\text { в }\end{array}$ & $\begin{array}{l}1279.00 \pm 17.35^{\mathrm{a}} \\
\text { A }\end{array}$ & $\begin{array}{l}1206.67 \pm 13.42^{\mathrm{a}} \\
\mathrm{C}\end{array}$ & $\begin{array}{l}1271.67 \pm 24.69^{\mathrm{a}} \\
\text { A }\end{array}$ & $\begin{array}{l}1246.75 \pm 14.49 \\
\text { a }\end{array}$ \\
\hline $\begin{array}{l}\text { Control } \\
(+\mathrm{ve}) \\
\text { (Infected } \\
\text { treated) }\end{array}$ & $649.00 \pm 14.18^{\mathrm{cA}}$ & $628.67 \pm 14.66^{\mathrm{fB}}$ & $\begin{array}{l}635.00 \pm 13.11^{\mathrm{fA}} \\
\mathrm{B}\end{array}$ & $623.67 \pm 14.68^{\mathrm{fB}}$ & $634.08 \pm 6.69^{f}$ \\
\hline $\begin{array}{l}\text { Exp. } \\
\text { infected }\end{array}$ & $614.00 \pm 10.02^{\mathrm{dB}}$ & $1191.33 \pm 7.86^{\mathrm{bA}}$ & $\begin{array}{l}1176.00 \pm 17.62^{b} \\
\text { A }\end{array}$ & $1183.33 \pm 4.26^{\mathrm{bA}}$ & $\begin{array}{l}1041.17 \pm 74.53 \\
\mathrm{~b}\end{array}$ \\
\hline
\end{tabular}


and

$\mathrm{ZnO}-$

NPs

treated

Exp.

infected

and nano $\quad 720.33 \pm 16.7^{\mathrm{bC}} \quad 913.00 \pm 11.36^{\mathrm{eA}} \quad 904.00 \pm 15.52^{\mathrm{eA}} \quad 845.33 \pm 6.57^{\mathrm{eB}} \quad 845.67 \pm 23.85^{\mathrm{e}}$ propolis

treated

a, b \& c: There is a significant difference $(\mathrm{P}<0.05)$ between any two means, within the same column have the different superscript letter.

A, B \& C: There is a significant difference $(\mathrm{P}<0.05)$ between any two means, within the same row have the different superscript letter.

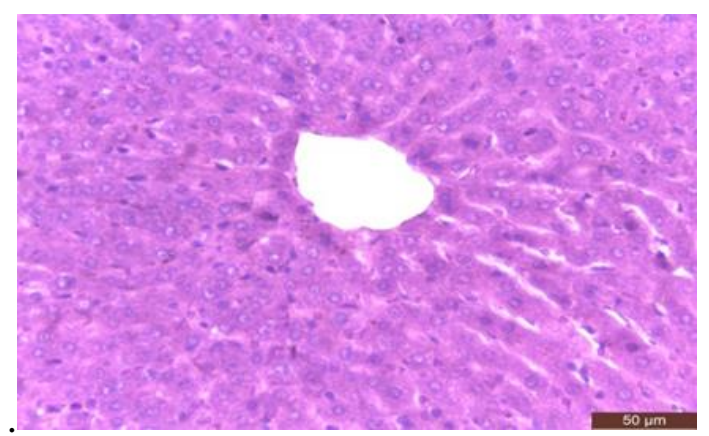

Fig.1. Liver from control negative group showing normal feature of histological liver structure; normal central vein, hepatocytes arranged in cords and separated blood sinusoid (H\&E stain, scale bar $=50 \mu \mathrm{m})$.

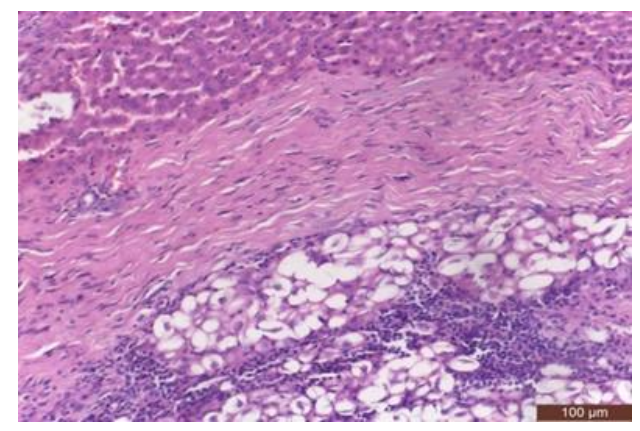

Fig.2. Liver from control positive group showing different protozoal stages. Notice thick C.T capsule surrounding the cyst (H\&E stain, scale bar $=100 \mu \mathrm{m})$.

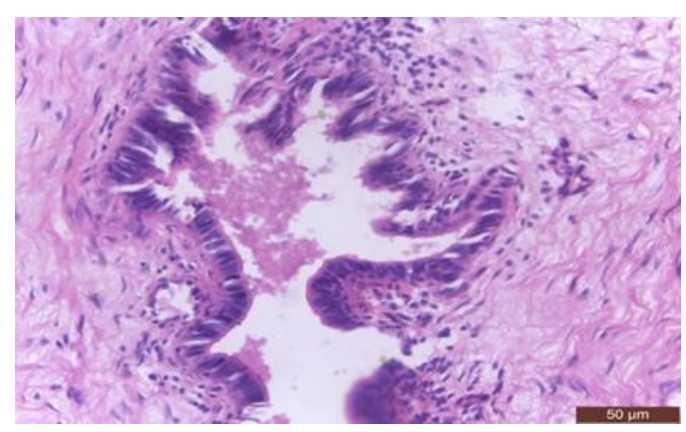


Fig.3. Liver from control positive group showing hyperplasia of biliary columnar epithelial cells $(\mathrm{H} \& \mathrm{E}$ stain, scale bar $=50 \mu \mathrm{m})$.

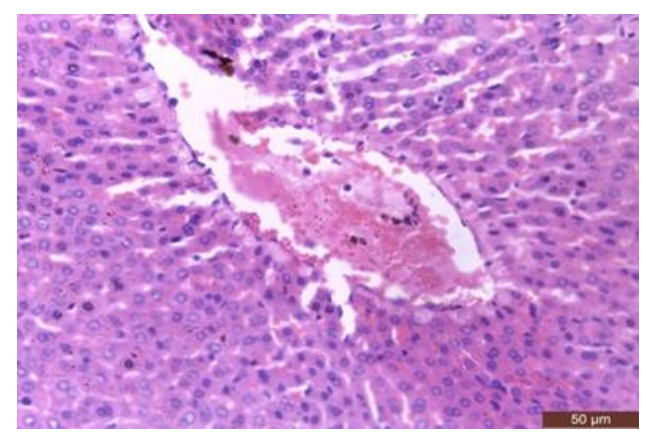

Fig.4. Liver from control positive group showing dilation and congestion of central vein with rupture of lining endothelial cells (H\&E stain, scale bar $=50 \mu \mathrm{m})$.

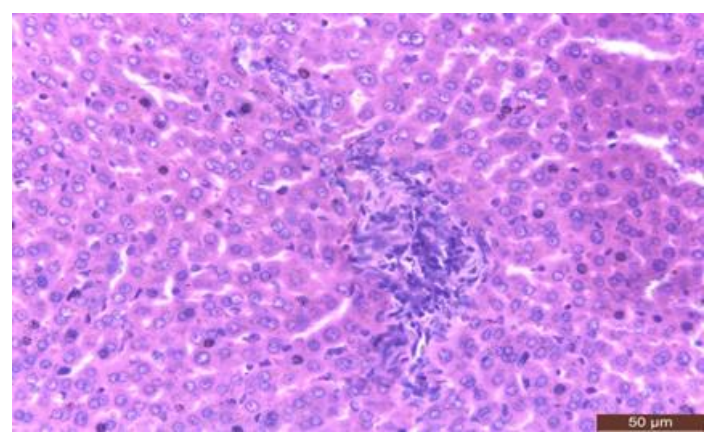

Fig.5. Liver from control positive group showing focal infiltration of mononuclear cells within hepatic parenchyma (H\&E stain, scale bar $=50 \mu \mathrm{m})$.

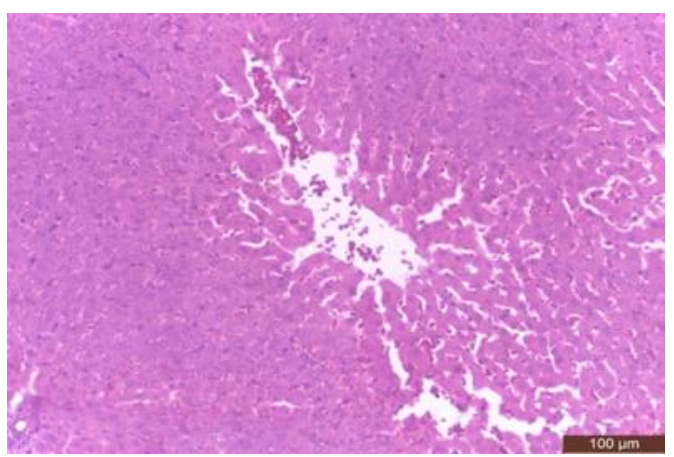

Fig.6. Liver from $\mathrm{ZnO}$-NPs treated group showing congestion of central vein and blood sinusoid (H\&E stain, scale bar $=100 \mu \mathrm{m})$

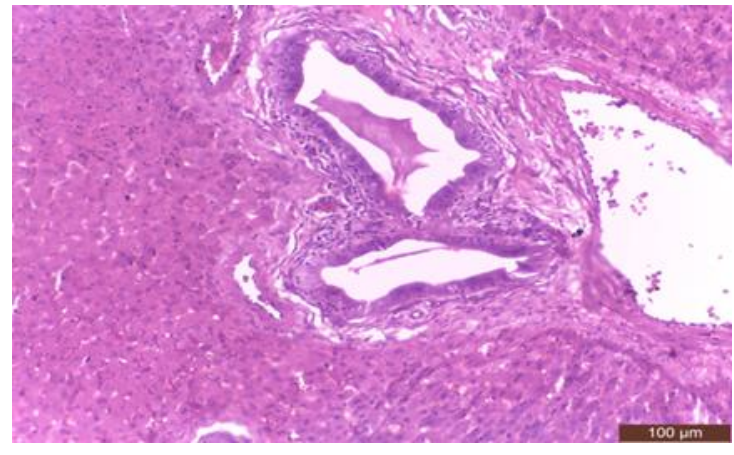

Fig.7. Liver from $\mathrm{ZnO}-\mathrm{NPs}$ treated group showing no papillary projection of epithelium of bile duct in comparison to control positive group. Also, notice decrease of peribiliary fibrosis (H\&E stain, scale bar $=100 \mu \mathrm{m})$. 


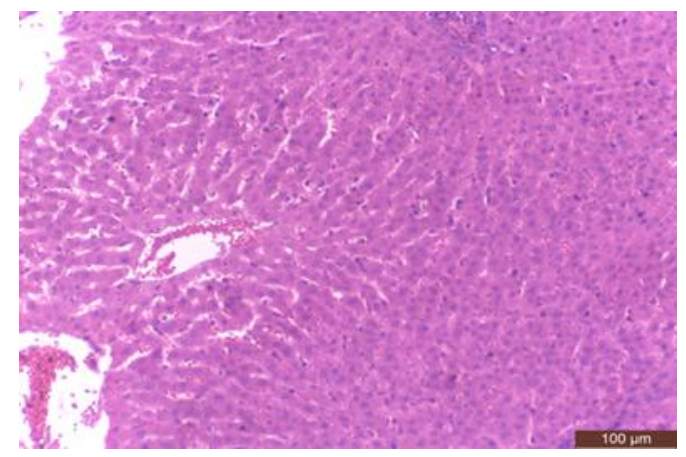

Fig.8. Liver from propolis-NPs treated group showing sever congestion of central vein and blood sinusoid (H\&E stain, scale bar $=100 \mu \mathrm{m})$.

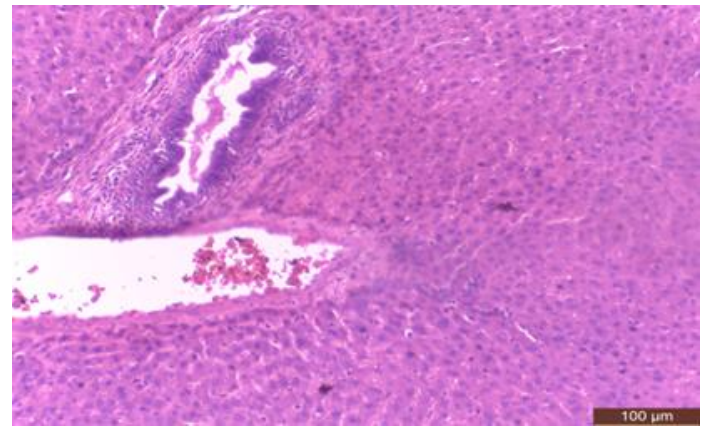

Fig.9. Liver from propolis-NPs treated group showing hyperplasia of epithelium of bile duct with fibrous coat (H\&E stain, scale bar $=100 \mu \mathrm{m})$.

\section{DISCUSSION}

Our results showed that ALT activity of infected rabbits with Eimeria spp. exhibited a significant increase, these results agreed with Cam et al. (2008) who reported that Eimeria stiedae in rabbits causes increasing of serum ALT activity compared to healthy control group. Administration of $\mathrm{ZnO}-\mathrm{NPs}$ to infected rabbits after 2 weeks of infection exhibited a significant decrease in serum ALT activity if compared to $\mathrm{ZnO}-\mathrm{NPs}$ infected group before treatment, these results agreed with Ahmadi et al.(2014) who reported that the dietary ZnO-NPs in broiler chicken showed decreasing serum alanine transferase (ALT). Administration of propolis-NPs to infected rabbits after 2 weeks of infection exhibited a significant decrease in ALT activity if compared to propolis-NPs infected group before treatment, these results agreed with Ambardekar et al. (2012) who reported that propolis by fabrication of liposomes has a platform nano-formulation for multicomponent natural medicine where formulation was able to suppress serum ALT activity in hepatotoxicity induced experimental animals and promote tissue healing.

In this study, cholinesterase activity exhibited a significant decrease; these results agreed with Jin et al. (2016) who recorded that cholinesterase were significantly different from the non-infected control. Administration of $\mathrm{ZnO}$-NPs to infected rabbits after 2 weeks of infection exhibited a significant increase in cholinesterase if compared to $\mathrm{ZnO}-\mathrm{NPs}$ infected group before treatment these results agreed with Bashandy et al.( 2017) who reported that thioacetamide (TAA) treatment led to a significant increase in liver enzymes, these parameters were reduced after treatment with $\mathrm{ZnO}-\mathrm{NPs}$. Administration of propolisNPs to infected rabbits after 2 weeks of infection exhibited a significant increase in 
cholinesterase if compared to propolis-NPs infected group before treatment these results agreed with Ambardekar et al.(2012) who reported that propolis by fabrication of liposomes has a platform nano-formulation has hepatoprotective properties.

The obtained data showed that serum CRP concentration of infected rabbits with Eimeria spp. exhibited a significant increase, these results agreed with Ragab et al .(2015) who reported that coccidiosis causes inflammation in tissues so levels of inflammatory mediators such as CRP showed increasing. Also, Pepys and Hirschfield. (2003) who declared that CRP is a sensitive systemic marker of inflammation and tissue damage. Administration of $\mathrm{ZnO}-\mathrm{NPs}$ to infected rabbits after 2 weeks of infection exhibited a significant decrease in CRP concentration if compared to $\mathrm{ZnO}-\mathrm{NPs}$ infected group before treatment these results agreed with Bashandy et al .(2017) who reported that thioacetamide (TAA) treatment led to a significant increase in plasma inflammatory markers but $\mathrm{ZnO}$ NPs decreased it . Administration of propolis -NPs to infected rabbits after 2 weeks of infection exhibited a significant decrease in CRP concentration if compared to propolis NPs infected group before treatment these results agreed with Nyun-Ki et al.(2010) who showed that the propolis -NPs was effective in the treatment of diabetes due to the reduction of blood sugar level and the regeneration of damaged $\beta$-cells observed in streptozotocin-induced diabetic rats so, nano propolis can decrease the inflammation of cells .

Our results showed that serum zinc concentration of infected rabbits with Eimeria spp. exhibited a significant decrease, these results agreed with Southern and Baker (1983) who reported that Liver $\mathrm{Zn}$ concentration was decreased by coccidiosis. Administration of $\mathrm{ZnO}-\mathrm{NPs}$ to infected rabbits after 2 weeks of infection exhibited a significant increase in zinc concentration if compared to ZnO-NPs infected group before treatment these results agreed with Hassan et al. (2017) who reported that diets of rabbits supplemented with zinc nanoparticles showed that the value of hepatic zinc increased. Administration of propolis-NPs to infected rabbits after 2 weeks of infection exhibited a significant increase in zinc concentration if compared to propolis-NPs infected group before treatment these results agreed with Lotfy( 2006) who reported that propolis contains minerals like zinc ( $\mathrm{Zn})$.

The obtained data showed that plasma GR, SOD and CAT activities of infected rabbits with Eimeria spp. exhibited a significant decrease, these results agreed with Kizil and Yuce (2009) who showed that coccidiosis in dogs revealed decreasing in GSH concentration and CAT activity. And Ghanem et al. (2009) who reported that there is a significant decrease in erythrocytes SOD, CAT and GSH concentration in Eimeria infected kids. Administration of ZnO-NPs to infected rabbits after 2 weeks of infection exhibited a significant increase in plasma GR, SOD and CAT activities if compared to $\mathrm{ZnO}$ NPs infected group before treatment these results agreed with (Atef et al., 2016) who declared that aflatoxicosis increased the concentration of NO and MDA, while decreased the level of GSH and activities of SOD, CAT and GPx. These changes were improved by administration of $\mathrm{ZnO}-\mathrm{NPs}$ to aflatoxicated animal through the immune strengthening effect and protection of lipid and protein from oxidative damage. Administration of propolis -NPs to infected rabbits after 2 weeks of infection exhibited a significant increase in plasma GR, SOD and CAT activities if compared to propolis -NPs infected group before treatment these results agreed with Hong-zhuan et al .(2008) who declared that propolis-NPs showed 
antioxidation activity where propolis nanometer could scavenge excessive free radicals in body better than common propolis and reduce the generation of superoxide lipids, which is a new anti-oxidation product. In conclusion, some nanoparticles such as $\mathrm{ZnO}-\mathrm{NPs}$ and propolis-NPs act as hepatoprotective and antioxidative agents against Eimeria infestation in rabbits.

\section{Histopathological of liver}

The liver of infected male rabbit with coccidiosis showing different stages of the protozoal stages that were agreed with those reported by Wang et al. (1991). Extensive hyperplasia of biliary columnar epithelial cells was identified that was agreed with Rosmini and Simoni (1979). Within the hepatic parenchyma, congestion of central vein and sinusoids like Al-Mathal (2008) and focal mononuclear cells infiltration like Singla et al. (2000) were recorded. The liver of infected $\mathrm{ZnO}$-NPs treated group showed no papillary projection of epithelium of bile duct with obvious decrease of peribiliary fibrosis were identified in comparison to control positive group. Also, there are no microgametocytes, macrogametocyts or oocyts in liver so $\mathrm{ZnO}$ NPs can decrease damaging of liver. These results were agreed with those reported by Bashandy et al. (2017) who reported the protective effect of $\mathrm{ZnO}-\mathrm{NPs}$.

The liver of propolis-NPs treated group, showed sever congestion of central vein and blood sinusoid without microgametocytes, macrogametocyts and oocyts in liver and liver showed hyperplasia of epithelium of bile duct with fibrous coat so propolis-NPs can decrease damaging of liver. These results were supported by finding of Nyun-Ki et al. (2010) and Hasan et al. (2016) who reported that the effective of propolis-NPs in the treatment of diabetes and regeneration of damaged cells and the treatment of rat mammary gland tumors respectively.

\section{REFERENCES}

Aebi, H. 1984. Catalase in vitro methods in enzymology (Ed. BJ Willam). Academic Press, New York, 105, 121 126.

Afrouzan, H., Amirinia, C., Mirhadi , S.A. , Ebadollahi ,A., Vaseji , N. and Tahmasbi , G. 2012. Evaluation of antimicrobial activity of propolis and nanopropolis against Staphylococcus aureus and Candida albicans. African Journal of Microbiology Research, 6(2), 421-425.

Ahmadi, F., Ebrahimnezjad, Y., Ghiasi ghalehkandi, J. and Sis, N. M. 2014. The Effect of dietary zinc oxide nanoparticles on the antioxidant state and serum enzymes activity in broiler chickens during starter stage. Int. J. Biosci.; 3:29-35.

Al-Mathal , E.M., 2008. Hepatic coccidiosis of the domastic rabbit Oryctolagus cuniculus domesticus in Saudi Arabia. World Journal of Zoology3 (1):30-35.

Al-Mathal , E.M., 2010. Efficacy of commiphora molmol against hepatic coccidiosis (Eimeria stiedae) in the domestic rabbit. Journal of food Agriculture and Environment ; 8, 3/4; 1072-1080.

Allahverdiyev , A. M., Abamor, E.S., Bagirova, M., Ustundag, C.B., Kaya, C., Kaya, F. and Rafailovich, M. 2011. Antileishmanial effect of silver nanoparticles and their enhanced antiparasitic activity under ultraviolet 
light. Int $\mathrm{J}$ Nanomedicine; 6:27052714.

Ambardekar, R.V., Mahadik, K.R., Paradkar, A.R. and Harsulkar, A.M. 2012. Enhancement of hepatoprotective efficacy of propolis by fabrication of liposomes, as a platform nanoformulation for multi-component natural medicine. Curr Drug Deliv.; 9(5):477-86.

Angeli, E., Buzio, R., Firpo, G., Magrassi , R., Mussi ,V., Repetto, L. and Valbusa, U. 2008. Nanotechnology applications in medicine. Tumori; 94(2):206-215.

Atef, H.A., Mansour, M.K. , Ibrahim , E.M., El-Ahl , R.M., Al-Kalamawey, N.M., El Kattanand,Y.A. and Ali, M.A. 2016. Efficacy of zinc oxide nanoparticles and curcumin in amelioration the toxic effects in aflatoxicated rabbits. International Journal of Current Microbiology and Applied Sciences ISSN: 2319 -7706 Volume 5, Number 12, pp. 795-818.

Bancroft, J.D. and Marilyn, G. 2008. Theory and Practice of Histological Techniqes. $6^{\text {th }}$ ed. Oxford: Churchill Livingstone Elsevier, 123 - 126.

Bashandy, S.A.E., Alaamer, A., Moussa, S.A. and Omara, E. 2017. Role of zinc oxide nanoparticles in alleviating hepatic fibrosis and nephrotoxicity induced by thioacetamide in rats. Can $\mathrm{J}$ Physiol pharmacol. Volume 0, Number ja, https://doi.org/10.1139/cjpp- 0247.

Búfalo,M.C., Figueiredo, A.S., de Sousa, J.P., Candeias, J.M., Bastos, J.K. and Sforcin, J.M. 2009. Anti-poliovirus activity of Baccharis dracunculifolia and propolis by cell viability deterimination and real-time PCR. J Apple Microbiol.107 (5):1669-80.

Cam, Y., Atasever, A., Erslan, G., Kibar, M., Atalay, O., Beyaz, L., Inci, A. and Liman, C.B. 2008. Eimeria stiedae : Experimental infection in rabbits and the effect of treatment with toltrazuril and ivermectin. Elsevier Inc. Experimental Parasitology; 119(1):164-172.

Coudert, P., Licois, D. and Zonnekeyn , V. 2000. Epizootic rabbit entercolitis and coccidiosis: a criminal conspiracy.In Proc.: 7thWorld Rabbit Congress, Valence, Spain, 215-21.

Coutinho, A. 2012. Honeybee propolis extract in periodontal treatment: A clinical and microbiological study of propolis in periodontal treatment. Indian $\mathrm{J}$ Dent Res.; 23: 294.

Dkhil, M.A., Al-Quraishy, S. and Wahab, R. 2015. Anticoccidial and antioxidant activities of zinc oxide nanoparticles on Eimeria papillata induced infection in the jejunum. Int J Nanomedicine ;10:1961-8.

ElAkabawy,L.M.,Zayan,K.A.,Tantawy,A.A.a ndOmar,R.E.M 2004. Anticoccidial efficacy of propolis and toltrazuril against Eimeria stiedae in New Zealand white rabbit's. Zag Vet J.; 32(1):129-145.

Fisher, J.W. and Kelley, G.L. 1977. The sporulated oocyst of Eimeria colini sp .n. from the bobwhite quail, Colinus virginianus. J Parasitol.; 63(2):200202.

Ghanem, M.M., Mohamed, A.D.A. and Ramadan, M.Y. 2009. Clinical, biochemical and histopathological study on parasitic gastroenteritis 
associated with caprine coccidiosis : comparative effect of toltrazuril and propolis. Lucrări Științifice Medicină Vol.52 No. 11(1) pp.565580.

Goldberg, D.M. and Spooner, R.J. 1983. Methods of Enzymatic analysis (Bergmeyen H.V.E.d) $3^{\text {rd }}$ edn vol 3,pp 258- 265.

Gunalan, S., Sivaraj, R. and Rajendran, V. 2012. Green synthesized $\mathrm{ZnO}$ nanoparticles against bacterial and fungal pathogens. Progress in Natural Science: Materials International, Volume 22, Issue 6, Pages 693-700.

Hasan, A.E.Z., Jumali, Jaja, D.M.,Sunarti, T.C , Suparno, O. and Setiyono, A. 2016. Antibreast cancer activity of nanopropolis Indonesia on induced mammary gland tumor by DMBA in virgin Sprague-dawley rats. BIOTROPIA, Vol.23No. 1,: 35 - 41 .

Hassan, F.A.M., Mahmoud, R. and El-Araby, I.E. 2017. Growth Performance, Serum Biochemical, Economic Evaluation and IL6 Gene .Expression in Growing Rabbits Fed Diets Supplemented with Zinc Nanoparticles. Zigzag Veterinary Journal, Volume 45, Number 3, p. 238-249.

Henry, J.B. 1974. Clinical diagnosis and management by laboratory method. W.B. Saunders Co., Philadelphia,Pap . 361.

Hong-Zhuan, X., Qing, S. and Jian-jun, M.A. 2008. Study on the Anti-oxidation Activity of Nanometer Propolis. [J]; Journal of Anhui Agricultural Sciences. Issue: 9 . R285.

Jin , J., Chun, L., Xing, Z.S., Mei, J.Y., Cheng, W.L., Yan, S. H. and Xiang,
S.Y. 2016. Pathological and ultrastructural observations and liver analysis of Eimeria stiedai-infected rabbits. Source: Veterinary Parasitology; 223:165-172.

Johnsen and Eliasson , R . 1987. Evaluation of a commercially available kit for the colorimetric determination of zinc. International Journal of Andrology ,10 (2):435 - 440 .

Kizil , O. and Yuce, A. 2009. Oxidative stress in dogs with coccidiosis. Revue Med. Vet.160, 11, 495-499.

Long, L., Chen, J., Zhang, Y., Liang, X., Ni , H., Zhang, B., Yin , Y. 2017. Comparison of porous and nano zinc oxide for replacing high-dose Dietary regular zinc oxide in weaning piglets. PLoS One. ; 12(11): e0188587.

Long, P.L., Millard, B.J.J., Oyner, L.P. and Norton, C.C. 1976. Guid to laboratory techniques used in the study and diagnosis of avian coccidiosis .Folia Vet. Lat., 6:201-217.

Lotfy, M. 2006. Biological activity of bee propolis in health and disease.Asian Pacific Journal of Cancer Prevention.;7(1):22-31.

Macleod, C.M. and Avery, O.T.T. 1941. The occurrence during acute infections of a protein not present in the Blood III. Immunological properties of the $\mathrm{C}$ reactive protein and its differentiation from normal blood proteins.J.Exp.Med;73(2) 191-200.

Nishikimi , M., Roa , N.A. and Yogi, K . 1972. The occurrence of super oxide anion in the reaction of reduced phenazine methosulfate and molecular oxygen Biochem.Bioph. Res. Common., 46,849-854. 
Nyun-Ki, C.,Young-Chae, C. ,ChangSu,H.and Hee-Sung, K. 2010. Hypoglycemic effects of nano powder propolis on streptozotocin- induced diabetic rats. Korean J Vet Serv 33(2): 199-206.

Oncel ,T., Gulegen, E., Senlik, B. and Bakirci, S. 2011. Intestinal coccidiosis in angora rabbits (Oryctolaguscuniculus) cause by Eimeria intestinalis, Eimeria perforans and Eimeria coecicola. YYU Veteriner Fakultesi Dergisi, 22(1),2729.

Pepys, M.B. and Hirschfield, G.M. 2003. Creactive protein: A critical update. J.Clin.Investig., 111,1805-1812.

Potkonjak, N.I., Veselinović, D.S., Novaković, M.M., Gorjanović, S.Ž., Pezo, L.L. and Sužnjević, D.Ž. 2012. Antioxidant activity of propolis extracts from Serbia:A polarographic approach. Food Chem Toxicol ; 50 (10):3614 -8.

Prasad, A.S., Bao, B., Beck, F.W., Kucuk, O. and Sarkar, F.H. 2004. Antioxidant effect of zinc in humans. Free Radic Biol Med. 15; 37(8):1182-90.

Ragab, A.I., Mekkawy, M.M., El-Gamil, A. and Mohanny, K.M. 2015. Effect of Propolis Dressing Technique on the Healing of Septic Diabetic Foot Ulcers . IOSR Journal of Nursing and Health Science (IOSR-JNHS) Volume 4, Issue 4 Ver. II, PP 33-52.

Rangasamy , J. , Lakshmanan, V. K. , Chandrasekaran, R. and Nair, S. 2012. In vitro Anti-cancerous and Antimicrobial Activity of Indian Propolis Nanoparticles. ASP Journal of
Nanopharmaceutics and Drug Delivery Vol. 1, 1-7.

Rosmini, R. and Simoni, P. 1979. Histological and ultrastructural features of hepatic coccidiosis in rabbits. Revista di Coniglicolture 16: 31-36.

Singla, L.D., Juyal, P.D. and Sandhu, B.S. 2000. Pathology and therapy in naturally Eimeria stiedae - infected rabbits. J. Protozool . Res. 10, 185191.

Southern, L.L. and Baker, D.H. 1983. Eimeria acervulina infection and the zinccopper interrelationship in the chick. Poult Sci.; 62(2):401-4.

Wang ,J.S. and Tsai, S.F. 1991. Prevalence and pathological study on rabbit hepatic coccidiosis in Taiwan. Department of Veterinary Medicine, National Chung- Hsing University, Taichung, Taiwan, Republic of China. Proc Natl Sci Counc Repub China B. ; 15(4):240-3. 